\title{
Efficiency fluctuations in microscopic machines
}

\author{
Sreekanth K Manikandan, ${ }^{1}$ Lennart Dabelow ${ }^{2}$ Ralf Eichhorn,${ }^{3}$ and Supriya Krishnamurthy ${ }^{1}$ \\ ${ }^{1}$ Department of Physics, Stockholm University, \\ SE-10691 Stockholm, Sweden. \\ ${ }^{2}$ Fakultät für Physik, Universität Bielefeld, 33615 Bielefeld, Germany \\ ${ }^{3}$ Nordita, Royal Institute of Technology and Stockholm University, \\ Roslagstullsbacken 23, SE-106 91 Stockholm, Sweden
}

( Dated: April 16, 2019)

\begin{abstract}
Nanoscale machines are strongly influenced by thermal fluctuations, contrary to their macroscopic counterparts. As a consequence, even the efficiency of such microscopic machines becomes a fluctuating random variable. Using geometric properties and the fluctuation theorem for the total entropy production, a "universal theory of efficiency fluctuations" at long times, for machines with a finite state space, was developed in [Verley et al., Nat. Commun. 5, 4721 (2014); Phys. Rev. E 90, 052145 (2014)]. We extend this theory to machines with an arbitrary state space. Thereby, we work out more detailed prerequisites for the "universal features" and explain under which circumstances deviations can occur. We also illustrate our findings with exact results for two non-trivial models of colloidal engines.
\end{abstract}

Understanding the functioning of machines on the micro- or nanoscale is of great interest because of their role in biological systems and their numerous technological applications [1-7]. Their small size makes this task a challenge since thermal fluctuations strongly affect their operation. As a result, average values are no longer sufficiently informative, and fluctuations in heat, work, efficiency etc. must be taken into account. Stochastic thermodynamics [8] provides a convenient framework for analyzing such systems by extending the notions of classical (ensemble-based) thermodynamics to individual realizations of a given process.

Consider first a macroscopic heat engine operating cyclically between two reservoirs at different temperatures $T_{1}>T_{2}$ and performing work against an external load force. If $Q_{1}$ and $Q_{2}$ denote the average heat exchanged with the two reservoirs and $-W$ the (average) performed work, the Second Law implies that the efficiency,

$$
\eta=-W / Q_{1},
$$

is universally bounded from above by the reversible or Carnot efficiency $\eta_{\mathrm{C}}=1-T_{2} / T_{1}$.

The efficiency $\eta$ plays an equally pivotal role for microscopic machines; however, in these systems, due to thermal fluctuations, the value obtained in individual realizations can deviate significantly from the average behavior. We hence need to consider a distribution of efficiency values. Recently, in two seminal papers [9, 10], Verley, Willaert, Van den Broeck, and Esposito (VWVE) developed a "universal theory of efficiency fluctuations" for machines with a finite state space. By characterizing the long-time behavior of the efficiency fluctuations in terms of their large-deviation function $J(\eta)$ (see below for more details), they found that the macroscopic efficiency, defined as the ratio of average output and input powers, is the most likely and, for machines operating in a non-equilibrium steady state or under a time-symmetric periodic protocol, the reversible Carnot efficiency is the least likely one [11]. The VWVE theory has since been verified in numerous model systems with finite [12-19] but also infinite $[13,19-21]$ state spaces.

Nevertheless, there are a few examples of infinite state space systems at odds with the theory [22-25], in which the rate function $J(\eta)$ fails to be smooth and/or does not exhibit a unique maximum at the reversible efficiency. A clear understanding of why some systems with infinite state space obey the "universal" theory while others do not is lacking. In this Letter, we give detailed prerequisites for when the features of the VWVE theory are found and when they are violated. In doing so, we develop an extended general theory of efficiency fluctuations, unifying the VWVE theory with deviations observed in specific models. Two examples of analytically solvable machines $[24,26]$ serve as illustrations for our general findings.

We start by briefly summarizing the approach taken in the VWVE theory. For all systems, the total work $W$ and heat $Q_{1}$ (as well as $Q_{2}$ ) grow extensively with increasing operational time $\tau$. For microscopic systems they also naturally fluctuate due to thermal noise, leading to a distribution $p_{\tau}\left(q_{1}, w\right)$ for observing a heat absorption rate $q_{1}=Q_{1} / \tau$ (the input power) and an output power $-w=-W / \tau$ with average values $\left\langle q_{1}\right\rangle$ and $\langle-w\rangle$. Using the theory of large deviations [27], we can quantify the asymptotic decay of the probability $p_{\tau}\left(q_{1}, w\right)$ towards the delta-distribution peaked at $\left\langle q_{1}\right\rangle$ and $\langle w\rangle$ by the large deviation or rate function $I\left(q_{1}, w\right)$,

$$
p_{\tau}\left(q_{1}, w\right) \sim e^{-\tau I\left(q_{1}, w\right)} \quad(\tau \rightarrow \infty),
$$

where $I\left(q_{1}, w\right) \geq 0$ and $I\left(\left\langle q_{1}\right\rangle,\langle w\rangle\right)=0$. Similarly, the stochastic efficiency $\eta=-w / q_{1}$ will tend towards the macroscopic efficiency $\bar{\eta}=\langle-w\rangle /\left\langle q_{1}\right\rangle$. Again, we can describe this approach in terms of a rate function $J(\eta)$, 

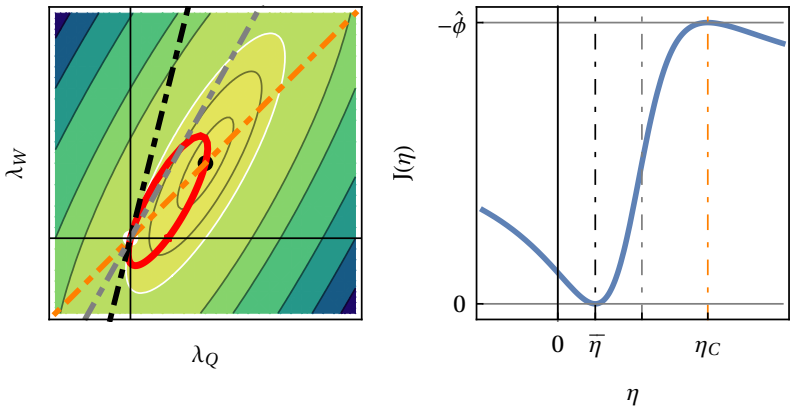

Figure 1. Illustration of the relation (5) between $\phi\left(\lambda_{Q}, \lambda_{W}\right)$ and $J(\eta)$. Left: Contour plot of $\phi\left(\lambda_{Q}, \lambda_{W}\right)$ showing its convexity along with the lines $\lambda_{Q}=\eta \lambda_{W}$ (dashed) for the macroscopic efficiency $\bar{\eta}$ (black), reversible efficiency $\eta_{\mathrm{C}}$ (orange) and an intermediate value (gray). The red curve marks the minimizing $\tilde{\boldsymbol{\lambda}}(\eta)$ for all $\eta$. Right: The resulting $J(\eta)=-\phi\left(\tilde{\lambda}_{Q}(\eta), \tilde{\lambda}_{W}(\eta)\right)$.

providing an asymptotic relation for the probability distribution $p_{\tau}(\eta)$,

$$
p_{\tau}(\eta) \sim e^{-\tau J(\eta)} \quad(\tau \rightarrow \infty) .
$$

This $J(\eta)$ can be extracted from the scaled cumulant generating function (sCGF) of heat and work $[9,10]$,

$$
\phi\left(\lambda_{Q}, \lambda_{W}\right):=\lim _{\tau \rightarrow \infty} \frac{1}{\tau} \ln \left\langle e^{-\lambda_{Q} Q_{1}-\lambda_{W} W}\right\rangle_{\tau},
$$

according to

$$
J(\eta)=-\min _{\lambda} \phi(\eta \lambda, \lambda) .
$$

Here $\langle\cdots\rangle_{\tau}$ denotes an average over the distribution $p_{\tau}\left(q_{1}, w\right)$. Note that $\phi\left(\lambda_{Q}, \lambda_{W}\right)$ is a convex function by definition.

The relation (5) implies $\phi(0,0)=0 \leq J(\eta) \leq-\hat{\phi}$, where $\hat{\phi}:=\min _{\lambda_{Q}, \lambda_{W}} \phi\left(\lambda_{Q}, \lambda_{W}\right)$ is the global minimum of $\phi$. Moreover, it has the geometric interpretation, illustrated in Fig. 1: For fixed $\eta$, We obtain $J(\eta)$ by minimizing $\phi\left(\lambda_{Q}, \lambda_{W}\right)$ along the line $\lambda_{Q}=\eta \lambda_{W}$ and inverting the sign. The set of all points $\tilde{\boldsymbol{\lambda}}(\eta) \equiv\left(\tilde{\lambda}_{Q}(\eta), \tilde{\lambda}_{W}(\eta)\right)$ where the minima are attained as a function of $\eta$ describes a curve in the $\left(\lambda_{Q}, \lambda_{W}\right)$-plane (see Fig. 1) with $J(\eta)=-\phi\left(\tilde{\lambda}_{Q}(\eta), \tilde{\lambda}_{W}(\eta)\right)$.

Exploiting this geometrical picture, the aforementioned "universal theory" by VWVE $[9,10]$ establishes generic properties of $J(\eta)$ that are independent of systemspecific details. As the main result they find that $J(\eta)$ is a smooth function with a unique minimum at the macroscopic efficiency $\bar{\eta}$, such that $J(\bar{\eta})=0$, and a unique maximum at some finite efficiency $\hat{\eta}$. For time-symmetric driving protocols, this "least likely" efficiency $\hat{\eta}$ coincides with the reversible efficiency $\eta_{\mathrm{C}}[11]$, see the example in Fig. 1.

These results of the VWVE theory are based on a few assumptions, most notably: (i) The detailed fluctuation theorem [28] $p\left(\Delta S_{\text {tot }}\right) / p\left(-\Delta S_{\text {tot }}\right)=\exp \left(\Delta S_{\text {tot }}\right)$ for the total entropy production $\Delta S_{\text {tot }}=-Q_{1} / T_{1}-Q_{2} / T_{2}+$ $\Delta S_{\text {sys }}$ (where $\Delta S_{\text {sys }}$ denotes the entropy change in the system itself) is valid, (ii) $\phi\left(\lambda_{Q}, \lambda_{W}\right)$ is a smooth function of its arguments and the fluctuation theorem implies that it has the symmetry property

$$
\phi\left(\lambda_{Q}, \lambda_{W}\right)=\phi\left(\lambda_{Q}^{*}-\lambda_{Q}, \lambda_{W}^{*}-\lambda_{W}\right)
$$

with $\lambda_{Q}^{*}=\eta_{\mathrm{C}} / T_{2}$ and $\lambda_{W}^{*}=1 / T_{2}$, and (iii) the minimum of $\phi\left(\lambda_{Q}, \lambda_{W}\right)$ is unique. The validity of (i) is by now wellestablished $[8,28]$. However, we will demonstrate that it does not necessarily entail the validity of the symmetry (6) for all $\left(\lambda_{Q}, \lambda_{W}\right)$ as in (ii). Further, we discuss the case that assumption (iii) does not hold either.

While assumption (ii) appears plausible for systems with finite state space, it has been observed in certain models with infinite state space that the sCGF (4) can have a restricted domain of convergence $C_{0}[22,24]$. It has also been noticed that the symmetry property (6) need not necessarily hold [24, 29-33]. To clarify the relationship between a limited convergence domain and the symmetry relation (6), we express the sCGF in terms of the individual time-extensive and -intensive contributions to the total entropy production,

$$
\Delta S_{\mathrm{tot}}=\frac{\eta_{\mathrm{C}}}{T_{2}} Q_{1}+\frac{1}{T_{2}} W+\Delta S_{\mathrm{int}} .
$$

Here, the term $\Delta S_{\text {int }}=-\frac{1}{T_{2}} \Delta E+\Delta S_{\text {sys }}$ collects the timeintensive contributions to the total entropy production that depend only on the initial and final states of the system. $\Delta E$ denotes the change in internal energy, which is, according to the First Law, $\Delta E=W+Q_{1}+Q_{2}$.

We first write down the moment-generating function (MGF) for the combined probability distribution of the individual contributions from (7),

$$
\Psi_{\tau}\left(\lambda_{Q}, \lambda_{W}, \lambda_{S}\right):=\left\langle e^{-\lambda_{Q} Q_{1}-\lambda_{W} W-\lambda_{S} \Delta S_{\text {int }}}\right\rangle_{\tau} .
$$

The fluctuation theorem for the total entropy production implies that $\Psi_{\tau}$ has the symmetry property [34,35], $\Psi_{\tau}\left(\lambda_{Q}, \lambda_{W}, \lambda_{S}\right)=\Psi_{\tau}\left(\lambda_{Q}^{*}-\lambda_{Q}, \lambda_{W}^{*}-\lambda_{W}, 1-\lambda_{S}\right)$. This symmetry is inherited by the three-dimensional sCGF

$$
\phi\left(\lambda_{Q}, \lambda_{W}, \lambda_{S}\right):=\lim _{\tau \rightarrow \infty} \frac{1}{\tau} \ln \Psi_{\tau}\left(\lambda_{Q}, \lambda_{W}, \lambda_{S}\right),
$$

so that

$$
\phi\left(\lambda_{Q}, \lambda_{W}, \lambda_{S}\right)=\phi\left(\lambda_{Q}^{*}-\lambda_{Q}, \lambda_{W}^{*}-\lambda_{W}, 1-\lambda_{S}\right) .
$$

The sCGF (4) of heat and work alone is the restriction of that "total" sCGF to the $\lambda_{S}=0$ plane, i.e. $\phi\left(\lambda_{Q}, \lambda_{W}\right)=$ $\phi\left(\lambda_{Q}, \lambda_{W}, 0\right)$.

As a consequence of Eq. (10) we arrive at the central observation that this restricted sCGF fulfills a "symmetry" relation of the form $\phi\left(\lambda_{Q}, \lambda_{W}\right)=\phi\left(\lambda_{Q}, \lambda_{W}, 0\right)=$ 


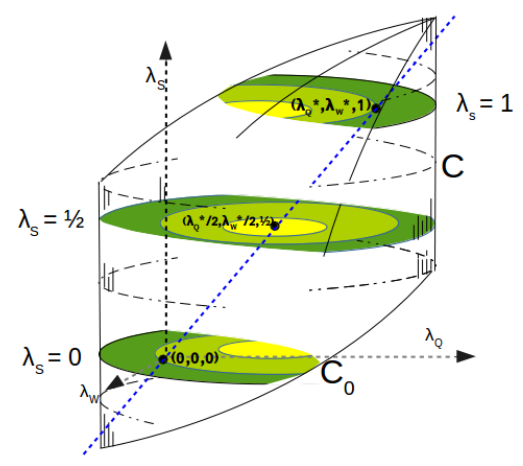

Figure 2. Contour plots of a typical $\phi\left(\lambda_{Q}, \lambda_{W}, \lambda_{S}\right)$ for $\lambda_{S}=$ $0, \frac{1}{2}, 1$ along with the domain of convergence $C$ and the domain of convergence $C_{0}$ for $\phi$ at $\lambda_{S}=0$. The functional form of $\phi\left(\lambda_{Q}, \lambda_{W}, \lambda_{S}\right)$ is the same in all $\lambda_{S}=$ const planes, but the limited domain of convergence leads to cutoffs whose location changes as a function of $\lambda_{S}$. The symmetry around the point $\left(\lambda_{Q}^{*} / 2, \lambda_{W}^{*} / 2,1 / 2\right)$ is a consequence of the fluctuation theorem.

$\phi\left(\lambda_{Q}^{*}-\lambda_{Q}, \lambda_{W}^{*}-\lambda_{W}, 1\right)$, instead of the relation (6). However, (6) could still be valid if $\phi\left(\lambda_{Q}, \lambda_{W}, \lambda_{S}\right)$ were independent of $\lambda_{S}$. Indeed, the VWVE theory $[9,10]$ is restricted to machines with a finite state space, in which case both, the internal energy $\Delta E$ and the system entropy $\Delta S_{\text {sys }}$, are bounded by $\tau$-independent constants, implying that the $\lambda_{S}$ dependence in (9) disappears in the $\tau \rightarrow \infty$ limit.

However, if fluctuations of the intensive entropy production $\Delta S_{\text {int }}$ can become arbitrarily large, as is typically the case for machines with infinite state space [24, 3641], we cannot argue that $\phi$ is independent of $\lambda_{S}$. In this case, too, the $\lambda_{S}$ contributions drop out in Eq. (9) as $\tau \rightarrow \infty$ wherever $\Psi_{\tau}\left(\lambda_{Q}, \lambda_{W}, \lambda_{S}\right)$ remains real and finite. However, in contrast to the finite state-space case, the limited domain of convergence $C$ of $\Psi_{\tau}$ will in general depend on $\lambda_{S}$. As a consequence of the fluctuation theorem symmetry obeyed by $\Psi_{\tau}, C$ is symmetric about the point $\left(\lambda_{Q}^{*} / 2, \lambda_{W}^{*} / 2,1 / 2\right)$. Therefore, the restriction of $C$ to the $\lambda_{S}=1 / 2$ plane satisfies a symmetry property like (6), but the domain of convergence $C_{0}$ of $\phi$ at $\lambda_{S}=0$ will in general not obey this symmetry, and hence neither will $\phi\left(\lambda_{Q}, \lambda_{W}, 0\right)$. This situation is illustrated in Fig. 2.

What are the consequences of the limited domain of convergence $C_{0}$ and its lack of symmetry for the large deviation function $J(\eta)$ ? The answer depends on whether the minimizing curve $\tilde{\boldsymbol{\lambda}}(\eta)$ is completely contained inside $C_{0}$ or whether it touches or hits the boundary of $C_{0}$. We illustrate this difference using the isothermal workto-work converter from Ref. [24], which exhibits both of these cases depending on the amplitude ratio of work and load forces. This machine consists of a Brownian particle in contact with a single heat bath at temperature $T$ and two additional white-noise forces, interpreted as a load and drive force, respectively. Identifying the work done by the drive force with $Q_{1}$ and the work done by the load force with $W$, we can calculate the $\operatorname{sCGF} \phi\left(\lambda_{Q}, \lambda_{W}\right)$ exactly, and from that the curve $\tilde{\boldsymbol{\lambda}}(\eta)$ and rate function $J(\eta)$ (see [24] and the Supplemental Material [42] which includes references $[43,44]$ for details).

In the first case, when $\tilde{\boldsymbol{\lambda}}(\eta)$ lies completely inside $C_{0}$, the existence of singular points for $\phi\left(\lambda_{Q}, \lambda_{W}\right)$ is irrelevant, resulting in a $J(\eta)$ that has exactly the properties and "universal shape" predicted by the VWVE theory (see the top panels in Fig. 3). In particular, the reversible efficiency $\eta_{\mathrm{C}}=1$ is still least likely, because the global minimum $\hat{\phi}$ of $\phi$ is still attained at the point $\left(\lambda_{Q}^{*} / 2, \lambda_{W}^{*} / 2\right)$ despite the "asymmetry" of $C_{0}$. By contrast, in the second case, $\phi$ takes its minimal value on the boundary of $C_{0}$ (lower left panel in Fig. 3). The minimizing curve $\tilde{\boldsymbol{\lambda}}(\eta)$ thus follows the boundary of $C_{0}$ for some range of $\eta$ values and becomes non-smooth at the points where the path transitions from the interior to the boundary and vice versa, leading to kinks in the first derivative of $J(\eta)$ (lower right panel in Fig. 3). We conclude that the appearance of cutoffs in $\phi\left(\lambda_{Q}, \lambda_{W}\right)$ can lead to discontinuities or "kinks" in $J(\eta)$ or its derivatives. In general (see also [42]), $J(\eta)$ is a smooth function of $\eta$ if and only if $\phi\left(\lambda_{Q}, \lambda_{W}\right)$ is smooth along the curve $\tilde{\boldsymbol{\lambda}}(\eta)$. Note that in the second example of Fig. 3 (lower panels), the least likely efficiency is still $\eta_{\mathrm{C}}$, even though $\phi\left(\lambda_{Q}, \lambda_{W}\right)$ does not obey the symmetry (6). However, this need not be the case in general since the minimal $\phi$ value need no longer be located on the line $\lambda_{Q}=\eta_{\mathrm{C}} \lambda_{W}$.

Next, we investigate the situation when assumption (iii) fails to hold and the global minimum of $\phi\left(\lambda_{Q}, \lambda_{W}\right)$ is not unique but rather degenerate [45], i.e. there exist multiple points $\left(\lambda_{Q}, \lambda_{W}\right)$ in the set $\hat{R}:=$ $\left\{\left(\lambda_{Q}, \lambda_{W}\right) \mid \phi\left(\lambda_{Q}, \lambda_{W}\right)=\hat{\phi}\right\}$. Due to the convexity of $\phi\left(\lambda_{Q}, \lambda_{W}\right)$, this set will be a connected region in the $\left(\lambda_{Q}, \lambda_{W}\right)$-plane. Then $J(\eta)$ assumes its maximal value $-\hat{\phi}$ for all $\eta$ for which the line $\lambda_{Q}=\eta \lambda_{W}$ intersects the region $\hat{R}$, leading to a plateau of degenerate maxima. Presumably, such a scenario could also occur in systems with finite state space. The reversible efficiency $\eta_{\mathrm{C}}$ is one of these maximizing efficiencies if and only if the line $\lambda_{Q}=\eta_{\mathrm{C}} \lambda_{W}$ intersects the region $\hat{R}$ within the domain of convergence $C_{0}$ (see also [42]).

We illustrate this situation with the example of the "Brownian gyrator" [26, 46]. This heat engine consists of a colloidal particle in two dimensions, immersed in a fluid environment and experiencing thermal fluctuations of different intensity along two perpendicular directions (temperatures $T_{1}$ and $T_{2}$, friction coefficients $\gamma_{1}$ and $\gamma_{2}$; see $[47,48]$ for experimental realizations). The particle is trapped in a harmonic potential whose principal axes with stiffnesses $u_{1}$ and $u_{2}$ are rotated by an angle $\alpha$ with respect to the preferred axes of the heat baths. 

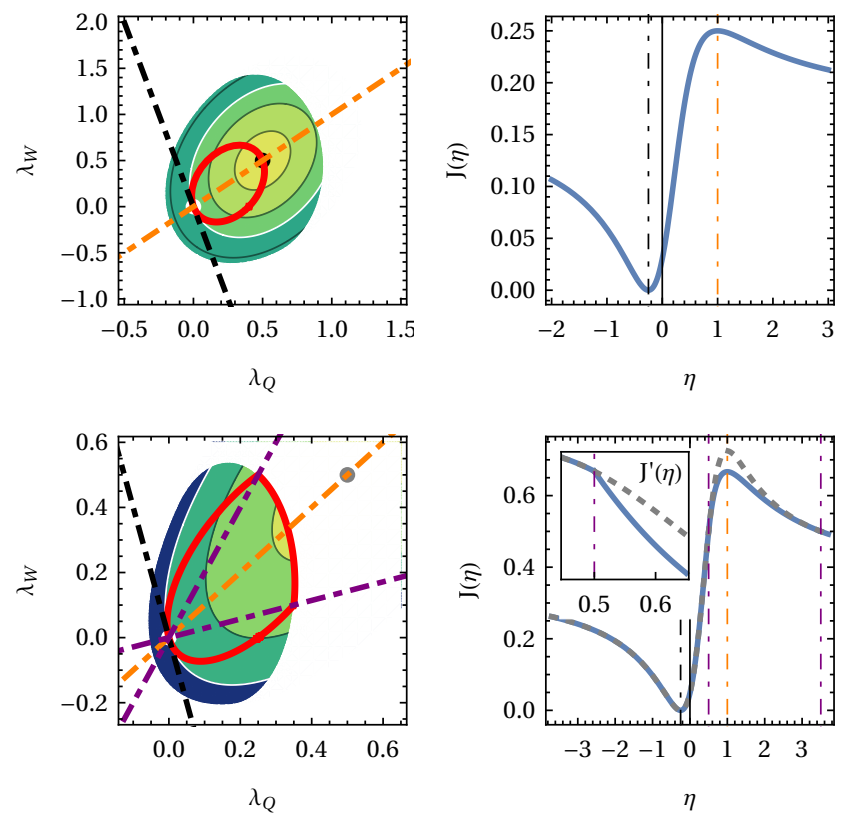

Figure 3. $\phi\left(\lambda_{Q}, \lambda_{W}\right)$ (left) and $J(\eta)$ (right) for the isothermal work-to-work converter from Ref. [24]. Exact results are shown for two different parameter sets of the model (see [24, 42] for details). Dashed black lines mark the corresponding macroscopic efficiencies $\bar{\eta}$ (negative in this case), dashed orange lines the reversible efficiency $\eta_{\mathrm{C}}=1$. Top (model parameters $\theta=1, \alpha=1 / 2$ ): Since the cutoff does not intersect the minimizing curve $\tilde{\boldsymbol{\lambda}}(\eta)$, the emerging shape of the rate function $J(\eta)$ still follows the predictions of the VWVE theory: It is smooth and has a unique maximum at the reversible efficiency $\eta_{\mathrm{C}}=1$. Bottom $(\theta=4, \alpha=1 / 2)$ : The cutoff interferes with the minimizing curve $\tilde{\boldsymbol{\lambda}}(\eta)$ so that the latter runs along the boundary of the domain of convergence for $\eta \in\left[\frac{1}{2}, \frac{7}{2}\right]$ (purple, dashed lines) and the rate function (right panel, blue curve) becomes distorted from the shape that would be obtained without cutoffs (dotted, gray curve). In particular, it develops kinks that become visible in its first derivative. One of these is displayed in the inset of the bottom-right panel.

As a consequence, the particle experiences a net torque letting it rotate around the origin on average [26]. Applying a linear "load torque" with slope $f_{\text {ext }}$, the system operates as a stationary, heat engine [46] (see also [42] for details). The resulting sCGF $\phi\left(\lambda_{Q}, \lambda_{W}\right)$ and rate function $J(\eta)$ can be computed exactly using path-integral techniques $[49,50]$ ( see also [42], which includes references [51-54]) and are shown for two different configurations in Fig. 4. In this system, the degenerate minimum of $\phi\left(\lambda_{Q}, \lambda_{W}\right)$ results from $\phi\left(\lambda_{Q}, \lambda_{W}\right)$ becoming a function of only $\lambda_{Q}-\bar{\eta} \lambda_{W}$ within the domain of convergence $C_{0}$ due to tight coupling between work and heat [14]. The iso-contours of $\phi$, one of them being the set $\hat{R}$, are therefore parallel lines with slope $1 / \bar{\eta}$ (see left panels of Fig. 4). The resulting plateaus for $J(\eta)$ are visible in the right panels. For the configuration in the
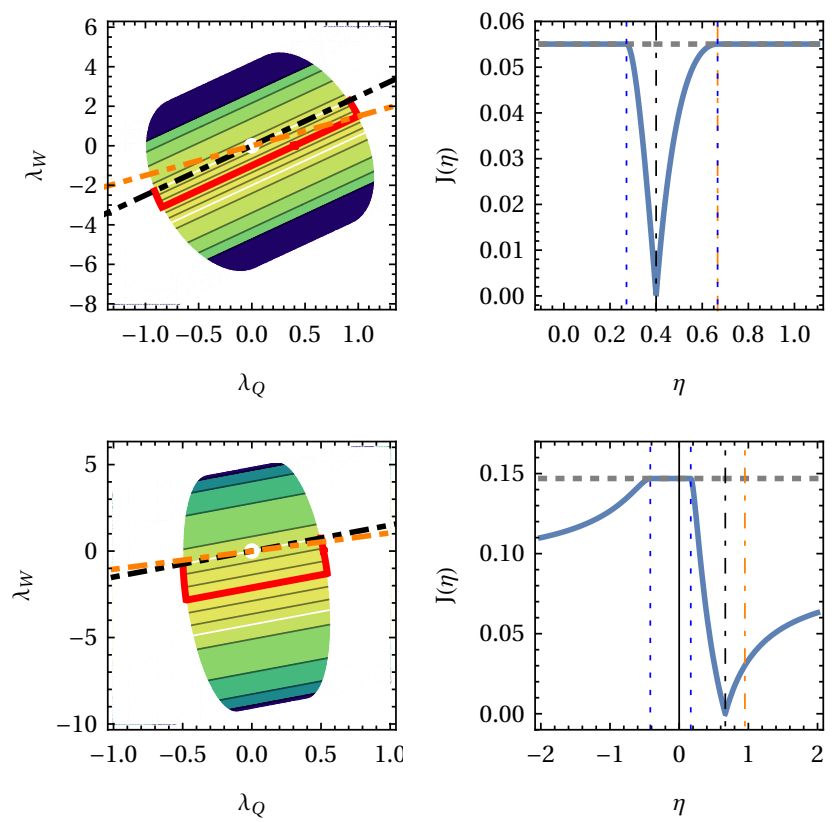

Figure 4. $\phi\left(\lambda_{Q}, \lambda_{W}\right)$ (left) and $J(\eta)$ (right) for two configurations of the Brownian gyrator. Dashed black lines mark the macroscopic efficiency $\bar{\eta}$, dashed orange lines the reversible efficiency $\eta_{\mathrm{C}}$. The minimizing curve $\tilde{\boldsymbol{\lambda}}(\eta)$ is shown in red in the left panels. The efficiencies at the edges of the plateau region are marked by dotted blue lines in the right panels. Top: $u_{1}=5, u_{2}=1, \alpha=\pi / 4, f_{\text {ext }}=-1 / 2, k_{\mathrm{B}} T_{1}=1$, $k_{\mathrm{B}} T_{2}=1 / 3$. Bottom: $u_{1}=4, u_{2}=2, \alpha=\pi / 4, f_{\text {ext }}=-1 / 2$, $k_{\mathrm{B}} T_{1}=2, k_{\mathrm{B}} T_{2}=1 / 10$. In all plots, $\gamma_{1}=\gamma_{2}=1$. In this case, the kinks occur in $J(\eta)$ itself.

top panels, the region $\hat{R}$ intersects the $\lambda_{Q}$-axis, so that the plateau of $J(\eta)$ extends to $\pm \infty$. Moreover, in this configuration the line $\lambda_{Q}=\eta_{\mathrm{C}} \lambda_{W}$ intersects $\hat{R}$, so that the Carnot efficiency lies at the edge of the plateau of degenerate maxima of $J(\eta)$. In contrast, for the second configuration in the lower panels, $\hat{R}$ does not intersect the $\lambda_{Q}$-axis and the plateau is restricted to a finite region of $\eta$ values. Furthermore, this plateau does not contain the Carnot efficiency $\eta_{\mathrm{C}}$. We note that in both cases $J(\eta)$ has kinks resulting from the minimizing curve $\tilde{\boldsymbol{\lambda}}(\eta)$ hitting the boundary of the domain of convergence $C_{0}$. A similar efficiency distribution has also been obtained in [22] for a closely related model.

In conclusion, we have extended the VWVE theory of efficiency fluctuations by including three crucial insights: First, the domain of convergence $C_{0}$ of $\phi\left(\lambda_{Q}, \lambda_{W}\right)$ determines if the fluctuation theorem symmetry (6) holds or not. The resulting cutoffs lead to a $J(\eta)$ differing from the VWVE theory, if and only if $\phi$ is non-smooth along the curve $\tilde{\boldsymbol{\lambda}}(\eta)$. Since $C_{0}$ depends on the boundary terms, this opens up possibilities to fine-tune initial conditions to change $J(\eta)$ and, for example, minimize fluctuations around the macroscopic efficiency. Secondly, $\phi$ can have degenerate minima, typically leading to a plateau 
of maximal values for $J(\eta)$.Finally, the symmetry $(6)$ is sufficient, but not necessary for the least likeliness of the Carnot efficiency $\eta_{\mathrm{C}}$. On the other hand, $\eta_{\mathrm{C}}$ is no longer least likely in general. Our results are obtained from generic features of the sCGF $\phi$ and incorporate as special cases the VWVE theory as well as exceptions found in specific models. Exact solutions for two non-trivial models support our observations.

Acknowledgements. LD acknowledges funding by the Deutsche Forschungsgemeinschaft within the Research Unit FOR 2692 under Grant No. 397303734 as well as by the Stiftung der Deutschen Wirtschaft. LD also thanks Nordita for the hospitality and support during an internship in 2016. RE acknowledges financial support from the Swedish Research Council (Vetenskapsrådet) under the grant No. 2016-05412.

[1] Jonathon Howard, Mechanics of Motor Proteins and the Cytoskeleton (Oxford University Press, 2001).

[2] JMR Parrondo and B Jiménez de Cisneros, "Energetics of Brownian motors: a review," Applied Physics A 75, 179-191 (2002).

[3] Giuliano Benenti, Giulio Casati, Keiji Saito, and Robert S Whitney, "Fundamental aspects of steady-state conversion of heat to work at the nanoscale," Phys. Rep. 694, 1-124 (2017).

[4] R Dean Astumian, "Microscopic reversibility as the organizing principle of molecular machines," Nat. Nanotechnol. 7, 684 (2012).

[5] Valentin Blickle and Clemens Bechinger, "Realization of a micrometre-sized stochastic heat engine," Nat. Phys. 8, 143 (2012).

[6] L Dinis, I A Martínez, É Roldán, J M R Parrondo, and R A Rica, "Thermodynamics at the microscale: from effective heating to the brownian carnot engine," Journal of Statistical Mechanics: Theory and Experiment 2016, 054003 (2016).

[7] Sudeesh Krishnamurthy, Subho Ghosh, Dipankar Chatterji, Rajesh Ganapathy, and A. K. Sood, "A micrometre-sized heat engine operating between bacterial reservoirs," Nature Physics 12, 1134 (2016).

[8] Udo Seifert, "Stochastic thermodynamics, fluctuation theorems and molecular machines," Rep. Prog. Phys. 75, 126001 (2012).

[9] Gatien Verley, Massimiliano Esposito, Tim Willaert, and Christian Van den Broeck, "The unlikely carnot efficiency," Nat. Commun. 5, 4721 (2014).

[10] Gatien Verley, Tim Willaert, Christian Van den Broeck, and Massimiliano Esposito, "Universal theory of efficiency fluctuations," Physical Review E 90, 052145 (2014).

[11] For machines driven asymmetrically in time, the reversible efficiency stands out as the value of $\eta$ at which the rate functions $J(\eta)$ and $\tilde{J}(\eta)$ for the forward and time-reversed drivings, respectively, intersect.

[12] Todd R Gingrich, Grant M Rotskoff, Suriyanarayanan Vaikuntanathan, and Phillip L Geissler, "Efficiency and large deviations in time-asymmetric stochastic heat en- gines," New J. Phys. 16, 102003 (2014).

[13] Karel Proesmans and Christian Van den Broeck, "Stochastic efficiency: five case studies," New Journal of Physics 17, 065004 (2015), arXiv:1503.00497 [condmat.stat-mech].

[14] M. Polettini, G. Verley, and M. Esposito, "Efficiency statistics at all times: Carnot limit at finite power," Phys. Rev. Lett. 114, 050601 (2015).

[15] Karel Proesmans, Cedric Driesen, Bart Cleuren, and Christian Van den Broeck, "Efficiency of single-particle engines," Phys. Rev. E 92, 032105 (2015).

[16] Massimiliano Esposito, Maicol A. Ochoa, and Michael Galperin, "Efficiency fluctuations in quantum thermoelectric devices," Phys. Rev. B 91, 115417 (2015).

[17] Gregory Bulnes Cuetara and Massimiliano Esposito, "Double quantum dot coupled to a quantum point contact: a stochastic thermodynamics approach," New J. Phys. 17, 095005 (2015).

[18] Jian-Hua Jiang, Bijay Kumar Agarwalla, and Dvira Segal, "Efficiency statistics and bounds for systems with broken time-reversal symmetry," Phys. Rev. Lett. 115, 040601 (2015).

[19] Bijay Kumar Agarwalla, Jian-Hua Jiang, and Dvira Segal, "Full counting statistics of vibrationally assisted electronic conduction: Transport and fluctuations of thermoelectric efficiency," Phys. Rev. B 92, 245418 (2015).

[20] K. Proesmans, B. Cleuren, and C. Van den Broeck, "Stochastic efficiency for effusion as a thermal engine," EPL (Europhysics Letters) 109, 20004 (2015).

[21] Marc Suñe and Alberto Imparato, "Efficiency fluctuations in steady-state machines," Journal of Physics A: Mathematical and Theoretical (2018).

[22] Jong-Min Park, Hyun-Myung Chun, and Jae Dong Noh, "Efficiency at maximum power and efficiency fluctuations in a linear brownian heat-engine model," Phys. Rev. E 94, 012127 (2016).

[23] Deepak Gupta, "Exact distribution for work and stochastic efficiency of an isothermal machine," J. Stat. Mech: Theory Exp. 2018, 073201 (2018).

[24] Deepak Gupta and Sanjib Sabhapandit, "Stochastic efficiency of an isothermal work-to-work converter engine," Phys. Rev. E 96, 042130 (2017).

[25] Hadrien Vroylandt, Thermodynamics and fluctuations of small machines, Theses, Université Paris-Saclay (2018).

[26] Roger Filliger and Peter Reimann, "Brownian gyrator: A minimal heat engine on the nanoscale," Phys. Rev. Lett. 99, 230602 (2007).

[27] Hugo Touchette, "The large deviation approach to statistical mechanics," Phys. Rep. 478, 1 - 69 (2009).

[28] U. Seifert, "Entropy production along a stochastic trajectory and an integral fluctuation theorem," Phys. Rev. Lett. 95, 040602 (2005).

[29] R. van Zon and E. G. D. Cohen, "Extended heatfluctuation theorems for a system with deterministic and stochastic forces," Phys. Rev. E 69, 056121 (2004).

[30] Jae Dong Noh, "Fluctuations and correlations in nonequilibrium systems," Journal of Statistical Mechanics: Theory and Experiment 2014, P01013 (2014).

[31] J. S. Lee, Chulan Kwon, and Hyunggyu Park, "Everlasting initial memory threshold for rare events in equilibration processes," Phys. Rev. E 87, 020104(R) (2013).

[32] Sanjib Sabhapandit, "Work fluctuations for a harmonic oscillator driven by an external random force," EPL (Europhysics Letters) 96, 20005 (2011). 
[33] Arnab Pal and Sanjib Sabhapandit, "Work fluctuations for a brownian particle in a harmonic trap with fluctuating locations," Phys. Rev. E 87, 022138 (2013).

[34] Joel L. Lebowitz and Herbert Spohn, "A gallavotticohen-type symmetry in the large deviation functional for stochastic dynamics," J. Stat. Phys. 95, 333-365 (1999).

[35] Reinaldo García-García, Daniel Domínguez, Vivien Lecomte, and Alejandro B. Kolton, "Unifying approach for fluctuation theorems from joint probability distributions," Phys. Rev. E 82, 030104(R) (2010).

[36] R. van Zon and E. G. D. Cohen, "Extended heatfluctuation theorems for a system with deterministic and stochastic forces," Phys. Rev. E 69, 056121 (2004).

[37] Jae Dong Noh, "Fluctuations and correlations in nonequilibrium systems," J. Stat. Mech: Theory Exp. 2014, P01013 (2014).

[38] J. S. Lee, Chulan Kwon, and Hyunggyu Park, "Everlasting initial memory threshold for rare events in equilibration processes," Phys. Rev. E 87, 020104(R) (2013).

[39] Gatien Verley, Christian Van den Broeck, and Massimiliano Esposito, "Work statistics in stochastically driven systems," New J. Phys. 16, 095001 (2014).

[40] Sanjib Sabhapandit, "Work fluctuations for a harmonic oscillator driven by an external random force," EPL (Europhysics Letters) 96, 20005 (2011).

[41] Arnab Pal and Sanjib Sabhapandit, "Work fluctuations for a brownian particle in a harmonic trap with fluctuating locations," Phys. Rev. E 87, 022138 (2013).

[42] See Supplemental Material at URL will be inserted by publisher.

[43] J. Gärtner, "On large deviations from the invariant measure," Theory Prob. Appl. 22, 24-39 (1977), http://dx.doi.org/10.1137/1122003.

[44] R. S. Ellis, "Large deviations for a general class of random vectors," Ann. Prob. 12, 1-12 (1984).

[45] Degeneracy in $\phi$ in systems with both tight coupling and dynamical phase transitions has been investigated in [25].

[46] Patrick Pietzonka and Udo Seifert, "Universal trade-off between power, efficiency, and constancy in steady-state heat engines," Phys. Rev. Lett. 120, 190602 (2018).

[47] Aykut Argun, Jalpa Soni, Lennart Dabelow, Stefano Bo, Giuseppe Pesce, Ralf Eichhorn, and Giovanni Volpe, "Experimental realization of a minimal microscopic heat engine," Physical Review E 96, 052106 (2017).

[48] K.-H. Chiang, C.-L. Lee, P.-Y. Lai, and Y.-F. Chen, "Electrical autonomous brownian gyrator," Phys. Rev. E 96, 032123 (2017).

[49] Sreekanth K. Manikandan and Supriya Krishnamurthy, "Asymptotics of work distributions in a stochastically driven system," The European Physical Journal B 90, 258 (2017).

[50] Sreekanth K Manikandan and Supriya Krishnamurthy, "Exact results for the finite time thermodynamic uncertainty relation," J. Phys. A: Math. Theor. 51, 11LT01 (2018).

[51] L. Onsager and S. Machlup, "Fluctuations and Irreversible Processes," Phys. Rev. 91, 1505-1512 (1953).

[52] S. Machlup and L. Onsager, "Fluctuations and Irreversible Process. II. Systems with Kinetic Energy," Phys. Rev. 91, 1512-1515 (1953).

[53] V. Y. Chernyak, M. Chertkov, and C. Jarzynski, "Path-integral analysis of fluctuation theorems for general Langevin processes," J. Stat. Mech. 2006, P08001 (2006).

[54] Klaus Kirsten and Alan J. McKane, "Functional determinants by contour integration methods," Ann. Phys. 308, 502-527 (2003)

\section{SUPPLEMENTAL MATERIAL}

This document provides further details of the calculations behind the results presented in the manuscript "Efficiency fluctuations in microscopic machines". In the first section, we further elaborate the relationship between properties of the scaled cumulant generating function of heat and work $\phi\left(\lambda_{Q}, \lambda_{W}\right)$ and properties of the efficiency rate function $J(\eta)$. In the second section, we summarize the essential findings (relevant for our analysis) of Ref. [24] about the isothermal work-to-work converter used as an illustrative example in the main text. In the third section, we introduce the Brownian gyrator model, which served as a second illustrative example in the main text, and present details on the derivation of its scaled cumulant generating function of heat and work.

\section{RELATION BETWEEN PROPERTIES OF $\phi\left(\lambda_{Q}, \lambda_{W}\right)$ AND PROPERTIES OF $J(\eta)$}

In this section, we formalize how certain prerequisites for the scaled cumulant generating function (sCGF) of heat and work $\phi\left(\lambda_{Q}, \lambda_{W}\right)$ lead to properties of the efficiency rate function $J(\eta)$, namely, smoothness, the least likely efficiency and plateaus.

Before we turn to the specific observations from the main text, we collect a few basic properties of the sCGF $\phi\left(\lambda_{Q}, \lambda_{W}\right)$. By definition (see Eq. (4) in the main text ), $\phi\left(\lambda_{Q}, \lambda_{W}\right)$ is a convex function, notably meaning that the sublevel sets

$$
A_{r}:=\left\{\left(\lambda_{Q}, \lambda_{W}\right) \in \mathbb{R}^{2}: \phi\left(\lambda_{Q}, \lambda_{W}\right) \leq r\right\}
$$

are convex. Moreover, it satisfies the normalization condition $\phi(0,0)=0$. As $J(\eta)$ is obtained from $\phi\left(\lambda_{Q}, \lambda_{W}\right)$ by 
minimizing along lines $\lambda_{Q}=\eta \lambda_{W}$ through the origin and inverting the sign (see Eq. (5) in the main text), this immediately implies $J(\eta) \geq 0$. It also implies that the curve $\tilde{\boldsymbol{\lambda}}(\eta)$ is contained in the sublevel set $A_{0}$.

Furthermore, for large deviation theory to be applicable to the problem at all, we need that the rate function $I\left(q_{1}, w\right)$, defined via Eq. (2) from the main paper, is well-defined and, in particular, $I(0,0)$ has a finite value. This in turn implies, according to the Gärtner-Ellis theorem $[27,43,44] I\left(q_{1}, w\right)=\max _{\lambda_{Q}, \lambda_{W}}\left[\lambda_{Q} q_{1}+\lambda_{W} w-\phi\left(\lambda_{Q}, \lambda_{W}\right)\right]$, that $\phi\left(\lambda_{Q}, \lambda_{W}\right)$ is bounded from below, so that there is a value $\hat{\phi} \in \mathbb{R}$ with $\phi\left(\lambda_{Q}, \lambda_{W}\right) \geq \hat{\phi}$ for all $\lambda_{Q}, \lambda_{W}$. All these properties will be taken for granted in the following.

\section{Smoothness}

As argued in the main text, $J(\eta)$ is a smooth function of $\eta$ if and only if $\phi\left(\lambda_{Q}, \lambda_{W}\right)$ is smooth along the curve $\tilde{\boldsymbol{\lambda}}(\eta)$. This follows immediately from the definition of $J(\eta)=-\min _{\lambda} \phi(\eta \lambda, \lambda)$ [see also Eq. (5) from the main paper] and from the definition of $\tilde{\boldsymbol{\lambda}}(\eta)$, implying $J(\eta)=-\phi\left(\tilde{\lambda}_{Q}(\eta), \tilde{\lambda}_{W}(\eta)\right)$. However, this criterion is not very "practical" because it generally becomes quite complicated to determine the curve $\tilde{\boldsymbol{\lambda}}(\eta)$ in the presence of singular points for $\phi\left(\lambda_{Q}, \lambda_{W}\right)$. A more accessible (albeit weaker) characterization is as follows:

If the global minimum of $\phi\left(\lambda_{Q}, \lambda_{W}\right)$ is unique and there exists an open region $U \subseteq \mathbb{R}^{2}$ with $A_{0} \subseteq U$ such that $\phi\left(\lambda_{Q}, \lambda_{W}\right)$ is smooth in $U$ and the Hessian matrix of $\phi\left(\lambda_{Q}, \lambda_{W}\right)$ is positive definite in $U$, then $J(\eta)$ is smooth.

This provides a sufficient (but not necessary) condition on $\phi\left(\lambda_{Q}, \lambda_{W}\right)$ for $J(\eta)$ to be smooth. To prove this, assume that $\phi\left(\lambda_{Q}, \lambda_{W}\right)$ is smooth in some open region $U$ containing the sublevel set $A_{0}$. Moreover, assume that the Hessian matrix of $\phi\left(\lambda_{Q}, \lambda_{W}\right)$ is positive definite on $U$, implying that the function is strictly convex on $U$. Smoothness means that $\phi\left(\lambda_{Q}, \lambda_{W}\right)$ is infinitely differentiable for all $\left(\lambda_{Q}, \lambda_{W}\right) \in U$. For every $\eta$, denote by $\tilde{\boldsymbol{\lambda}}(\eta) \equiv\left(\tilde{\lambda}_{Q}(\eta), \tilde{\lambda}_{W}(\eta)\right)$ a point with $\tilde{\lambda}_{Q}(\eta)=\eta \tilde{\lambda}_{W}(\eta)$ that minimizes Eq. (5), i.e. $\phi\left(\tilde{\lambda}_{Q}(\eta), \tilde{\lambda}_{W}(\eta)\right)=\min _{\lambda} \phi(\eta \lambda, \lambda)$. As observed above, $J(\eta) \geq 0$ for all $\eta$, so that $\tilde{\boldsymbol{\lambda}}(\eta) \in A_{0} \subseteq U$. Obviously, $J(\eta)=-\phi\left(\tilde{\lambda}_{Q}(\eta), \tilde{\lambda}_{W}(\eta)\right)$, meaning that the function $J(\eta)$ is determined by the values of $\phi\left(\lambda_{Q}, \lambda_{W}\right)$ on the curve $\eta \mapsto \tilde{\boldsymbol{\lambda}}(\eta)$ with $\eta \in \mathbb{R}$. It suffices to show that this mapping is smooth. The smoothness of $\phi\left(\lambda_{Q}, \lambda_{W}\right)$ in $U$ then implies that $J(\eta)=-\phi\left(\tilde{\lambda}_{Q}(\eta), \tilde{\lambda}_{W}(\eta)\right)$ is smooth as well.

Smoothness and convexity of $\phi\left(\lambda_{Q}, \lambda_{W}\right)$ imply that the line $\lambda_{Q}=\eta \lambda_{W}$ is tangent to the iso-contour $\phi\left(\lambda_{Q}, \lambda_{W}\right)=$ $J(\eta)$ in the point $\tilde{\boldsymbol{\lambda}}(\eta)$. Due to smoothness, the $\phi\left(\lambda_{Q}, \lambda_{W}\right)=r$ iso-contour is $\partial A_{r}$, the boundary of the sublevel set $A_{r}$ from Eq. (11). Since $\phi\left(\lambda_{Q}, \lambda_{W}\right)$ is strictly convex, so are the sublevel sets $A_{r}$, and consequently the point $\tilde{\boldsymbol{\lambda}}(\eta)$ is unique for all $\eta$. The fact that the line of efficiency $\eta$ is tangent to an iso-contour of $\phi\left(\lambda_{Q}, \lambda_{W}\right)$ in $\tilde{\lambda}(\eta)$ means that the ray vector $(\eta, 1)$ of the line $\lambda_{Q}=\eta \lambda_{W}$ is orthogonal to the gradient of $\phi\left(\lambda_{Q}, \lambda_{W}\right)$ in $\tilde{\boldsymbol{\lambda}}(\eta)$. Thus

$$
\eta \frac{\partial \phi}{\partial \lambda_{Q}}\left(\tilde{\lambda}_{Q}(\eta), \tilde{\lambda}_{W}(\eta)\right)+\frac{\partial \phi}{\partial \lambda_{W}}\left(\tilde{\lambda}_{Q}(\eta), \tilde{\lambda}_{W}(\eta)\right)=0 .
$$

This relation along with $\tilde{\lambda}_{Q}(\eta)=\eta \tilde{\lambda}_{W}(\eta)$ implicitly defines $\tilde{\boldsymbol{\lambda}}(\eta)$ as a function of $\eta$. More precisely, we consider the function

$$
f(\eta, \lambda):=\eta \frac{\partial \phi}{\partial \lambda_{Q}}(\eta \lambda, \lambda)+\frac{\partial \phi}{\partial \lambda_{W}}(\eta \lambda, \lambda) .
$$

Assume that we have a particular solution $\lambda_{*}$ of (12) for some given $\eta_{*}$, so that $f\left(\eta_{*}, \lambda_{*}\right)=0$. Due to the assumed positive definiteness of the Hessian matrix of $\phi\left(\lambda_{Q}, \lambda_{W}\right)$, we have

$$
\frac{\partial f}{\partial \lambda}\left(\eta_{*}, \lambda_{*}\right)=\left(\begin{array}{ll}
\eta_{*} & 1
\end{array}\right)\left(\begin{array}{cc}
\frac{\partial^{2} \phi}{\partial \lambda_{Q}^{2}}\left(\eta_{*}, \lambda_{*}\right) & \frac{\partial^{2} \phi}{\partial \lambda_{Q} \partial \lambda_{W}}\left(\eta_{*}, \lambda_{*}\right) \\
\frac{\partial^{2} \phi}{\partial \lambda_{W} \partial \lambda_{Q}}\left(\eta_{*}, \lambda_{*}\right) & \frac{\partial^{2} \phi}{\partial \lambda_{Q}^{2}}\left(\eta_{*}, \lambda_{*}\right)
\end{array}\right)\left(\begin{array}{c}
\eta_{*} \\
1
\end{array}\right)>0 .
$$

By the implicit function theorem, there exists a function $\eta \mapsto \tilde{\lambda}(\eta)$ on an open interval $I \subseteq \mathbb{R}$ with $\eta_{*} \in I$ and such that $f(\eta, \tilde{\lambda}(\eta))=0$ for all $\eta \in I$. Moreover, this function is of the same differentiability class as $f(\eta, \lambda)$. Put differently, the parameter function $\eta \mapsto \tilde{\lambda}(\eta)$ implicitly defined by Eq. (12) is well-defined and smooth in $I$. As this holds everywhere in $U$, we conclude that $\tilde{\lambda}(\eta)=(\eta \tilde{\lambda}(\eta), \tilde{\lambda}(\eta))$ and thus $J(\eta)=-\phi\left(\tilde{\lambda}_{Q}(\eta), \tilde{\lambda}_{W}(\eta)\right)$ is smooth.

\section{Least likely efficiency}

The "least likely" efficiency, i.e. the value $\hat{\eta}$ that maximizes $J(\eta)$, is directly related to the global minimum of $\phi\left(\lambda_{Q}, \lambda_{W}\right)$. Indeed, if $\phi\left(\lambda_{Q}, \lambda_{W}\right)$ assumes its minimal value at $\left(\hat{\lambda}_{Q}, \hat{\lambda}_{W}\right)$, then $J(\eta)$ will become maximal for $\hat{\eta}=$ 
$\hat{\lambda}_{Q} / \hat{\lambda}_{W}$ by Eq. (5). As observed in the main text and investigated in more detail in the next section of this Supplemental Material, the global minimum of $\phi\left(\lambda_{Q}, \lambda_{W}\right)$ need not be unique in general, so that $J(\eta)$ can have a degenerate maximum ("plateau") as well. In any case, the reversible efficiency $\eta_{\mathrm{C}}$ maximizes $J(\eta)$ if and only if the global minimum of $\phi\left(\lambda_{Q}, \lambda_{W}\right)$ lies on the line $\lambda_{Q}=\eta_{\mathrm{C}} \lambda_{W}$.

As observed in Refs. [9, 10], the symmetry property Eq. (6) provides a sufficient (but not necessary) condition for the least likeliness of the reversible efficiency $\eta_{\mathrm{C}}$. Indeed, if Eq. (6) holds then the iso-contour lines of $\phi\left(\lambda_{Q}, \lambda_{W}\right)$ are invariant under reflection through the point $\left(\lambda_{Q}^{*} / 2, \lambda_{W}^{*} / 2\right)$. By convexity, $\phi\left(\lambda_{Q}, \lambda_{W}\right)$ must therefore attain its minimal value in the reflection point, so that $\phi\left(\lambda_{Q}^{*} / 2, \lambda_{W}^{*} / 2\right)=\hat{\phi}$. Hence $J(\eta)$ assumes the maximum possible value for the line with slope $\hat{\eta}=\lambda_{Q}^{*} / \lambda_{W}^{*}=\eta_{\mathrm{C}}$.

\section{Plateau}

We have illustrated in the main text that there could be cases where the global maximum of $J(\eta)$ is not unique, meaning that there may be an entire "plateau region" where $J(\eta)$ assumes its maximal value. As stated in the main text,

The maximum of $J(\eta)$ is unique if and only if all minimizing points $\left(\lambda_{Q}, \lambda_{W}\right)$ of $\phi$ lie on a line $\lambda_{Q}=\hat{\eta} \lambda_{W}$ through the origin with fixed slope $\hat{\eta}$.

Below, we elaborate on this feature.

Let us first assume that there exist $\boldsymbol{\lambda}^{(1)}, \boldsymbol{\lambda}^{(2)} \in A_{\hat{\phi}}$ with $\boldsymbol{\lambda}^{(1)} \neq \boldsymbol{\lambda}^{(2)}$ and $\lambda_{Q}^{(1)} / \lambda_{W}^{(1)} \neq \lambda_{Q}^{(2)} / \lambda_{W}^{(2)}$, so that the minimizing points of $\phi\left(\lambda_{Q}, \lambda_{W}\right)$ do not lie on a single line through the origin. (Recall that $A_{\hat{\phi}}$ denotes the set of all $\boldsymbol{\lambda}=\left(\lambda_{Q}, \lambda_{W}\right)$ with $\phi\left(\lambda_{Q}, \lambda_{W}\right)=\hat{\phi}$, c.f. Eq. (11)). The latter condition ensures that the slopes $\eta^{(i)}=\lambda_{Q}^{(i)} / \lambda_{W}^{(i)}$ of the lines connecting the origin with $\boldsymbol{\lambda}^{(1)}$ and $\boldsymbol{\lambda}^{(2)}$, respectively, are different, meaning that $\eta^{(1)} \neq \eta^{(2)}$. But since both lines cut through the global minimum, it follows that $J\left(\eta^{(1)}\right)=J\left(\eta^{(2)}\right)=-\hat{\phi}$, establishing the degeneracy of $J(\eta)$. Moreover, by convexity of $\phi\left(\lambda_{Q}, \lambda_{W}\right)$, all lines with slopes $\eta$ between $\eta^{(1)}$ and $\eta^{(2)}$ will also cross the global minimum, so that all plateau efficiencies are connected. In other words, there cannot be two separate plateaus in disjoint intervals of the extended(!) real line $\mathbb{R} \cup\{\infty\}$. (However, the plateau may be connected through the point $\eta= \pm \infty$, corresponding to the line $\lambda_{W}=0$.) We remark that the emergence of plateaus need not necessarily be due to "tight coupling" as in the Brownian gyrator example presented in the main text (Fig.4 ). The tight coupling case, where $\phi\left(\lambda_{Q}, \lambda_{W}\right)=\phi\left(\lambda_{Q}-\bar{\eta} \lambda_{W}\right)$ is a special case exhibiting a degenerate global minimum.

To show the converse direction, assume that there is a unique $\hat{\eta}$ such that all points $\hat{\lambda}=\left(\hat{\lambda}_{Q}, \hat{\lambda}_{W}\right)$ with $\phi\left(\hat{\lambda}_{Q}, \hat{\lambda}_{W}\right)=$ $\hat{\phi}$ satisfy $\hat{\lambda}_{Q} / \hat{\lambda}_{W}=\hat{\eta}$. Then all such points $\hat{\lambda}$ lie on the line $\lambda_{Q}=\hat{\eta} \lambda_{W}$, while for all $\eta \neq \hat{\eta}$ and all $\lambda \in \mathbb{R}, \phi(\eta \lambda, \lambda)>\hat{\phi}$. Hence $J(\eta)$ has a unique maximum at $\hat{\eta}$.

\section{EXAMPLE 1: ISOTHERMAL WORK-TO-WORK CONVERTER ENGINE [24]}

In this section, we provide details about the example of an isothermal work-to-work converter by briefly summarizing the main results from Ref. [24] that are relevant for our purposes.

The model consists of a Brownian particle of mass $m$ in a fluid environment at temperature $T$ with instantaneous velocity $v(t)$. By the fluctuation-dissipation theorem, the coupling to the heat bath gives rise to a fluctuating force $\sqrt{2 k_{\mathrm{B} T \gamma} \gamma} \eta(t)$ as well as a frictional force $-\gamma v(t)$, where $\gamma$ is the friction coefficient and $\eta(t)$ is a Gaussian white-noise process with $\langle\eta(t)\rangle=0$ and $\left\langle\eta(t) \eta\left(t^{\prime}\right)\right\rangle=\delta\left(t-t^{\prime}\right)$. In addition, the particle is subject to two more fluctuating forces $f_{1}(t)$ and $f_{2}(t)$ with Gaussian white-noise statistics, independent of each other as well as of the thermal noise, i.e. $\left\langle f_{i}(t) f_{j}\left(t^{\prime}\right)\right\rangle=\delta_{i j} \bar{f}_{i}^{2} \delta\left(t-t^{\prime}\right)$ and $\left\langle f_{i}(t) \eta\left(t^{\prime}\right)\right\rangle=0$. The resulting equation of motion thus reads

$$
m \dot{v}(t)=-\gamma v(t)+f_{1}(t)+f_{2}(t)+\sqrt{2 k_{\mathrm{B}} T \gamma} \eta(t)
$$

The relative strength of the three fluctuating forces with respect to each other is parameterized by the positive parameters $\theta$ and $\alpha$ such that $\bar{f}_{1}^{2}=2 k_{\mathrm{B}} T \gamma \theta$ and $\bar{f}_{2}^{2}=2 k_{\mathrm{B}} T \gamma \theta \alpha^{2}$. The force $f_{1}(t)$ and $f_{2}(t)$ are interpreted as a load and drive force, respectively. The work done by them is given by

$$
W_{i}=\frac{1}{T} \int_{0}^{\tau} d t f_{i}(t) v(t)
$$




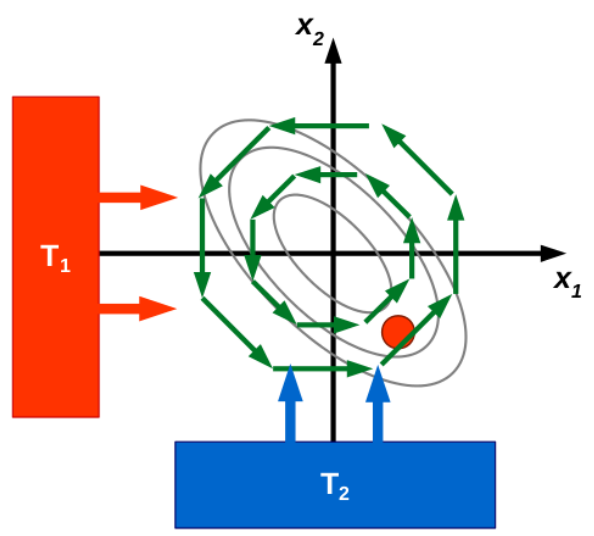

Figure 5. Sketch of the Brownian gyrator: A Brownian particle in two dimensions, sits in a potential $U$ (gray) and experiences an additional vortex force $\boldsymbol{f}_{\text {ext }}$ (green). It dissipates energy into a (fluid) medium via friction and is also subject to thermally fluctuating forces from collisions with the fluid molecules. The medium itself is in contact with two orthogonally irradiating reservoirs at temperatures $T_{1}$ (red) and $T_{2}$ (blue), leading to different fluctuation intensities in the two coordinate directions.

Translated to the setting in the main text, we thus identify $W_{1}$ with $W$ and $W_{2}$ with $Q_{1}$. The resulting momentgenerating function for $W_{1}$ and $W_{2}$ was found in Ref. [24] to satisfy the asymptotic relation

$$
\Psi_{\tau}\left(\lambda_{1}, \lambda_{2}\right)=\left\langle e^{-\lambda_{1} W_{1}-\lambda_{2} W_{2}}\right\rangle_{\tau} \sim g\left(\lambda_{1}, \lambda_{2}\right) e^{\tau \mu\left(\lambda_{1}, \lambda_{2}\right)} \quad(\tau \rightarrow \infty),
$$

where

$$
\begin{aligned}
\mu\left(\lambda_{1}, \lambda_{2}\right) & =\frac{1}{2}\left[1-\nu\left(\lambda_{1}, \lambda_{2}\right)\right], \\
\nu\left(\lambda_{1}, \lambda_{2}\right) & =\left[1+4 \theta\left\{\lambda_{1}\left(1-\lambda_{1}\right)+\alpha^{2} \lambda_{2}\left(1-\lambda_{2}\right)-\alpha^{2} \theta\left(\lambda_{1}-\lambda_{2}\right)^{2}\right\}\right]^{\frac{1}{2}}, \\
g\left(\lambda_{1}, \lambda_{2}\right) & =\sqrt{\frac{2 \sqrt{-4 \alpha^{2} \theta^{2}\left(\lambda_{1}-\lambda_{2}\right)^{2}-4 \theta\left(\alpha^{2}\left(\lambda_{2}-1\right) \lambda_{2}+\left(\lambda_{1}-1\right) \lambda_{1}\right)+1}}{\sqrt{-4 \alpha^{2} \theta^{2}\left(\lambda_{1}-\lambda_{2}\right)^{2}+4 \theta\left(\alpha^{2}\left(-\left(\lambda_{2}-1\right)\right) \lambda_{2}-\lambda_{1}^{2}+\lambda_{1}\right)+1}+2 \theta\left(-\lambda_{1}^{2}\left(\alpha^{2} \theta+\theta+1\right)+\alpha^{2}\left(-\lambda_{2}\right)\left(\lambda_{2}\left(\alpha^{2} \theta+\theta+1\right)-1\right)+\lambda_{1}\right)+1}} .
\end{aligned}
$$

From this, the scaled cumulant generating function $\phi\left(\lambda_{1}, \lambda_{2}\right) \equiv \phi\left(\lambda_{Q}, \lambda_{W}\right)$ can be extracted straightforwardly.

\section{EXAMPLE 2: BROWNIAN GYRATOR}

In this section, we give a detailed definition of the Brownian gyrator model adapted from Ref. [26] and provide the exact solution of its scaled cumulant generating function $\phi\left(\lambda_{Q}, \lambda_{W}\right)$.

The model consists of a Brownian particle in two dimensions at position $\mathbf{x}=\left(x_{1} x_{2}\right)^{T}$, sketched in Fig. 5 . The particle is immersed in a fluid environment and simultaneously coupled to two (effective) heat baths at different temperatures $T_{1}>T_{2}$ that only act in the $x_{1}$ and $x_{2}$ directions, respectively. For example, the colder temperature $T_{2}$ may be the temperature of the surrounding fluid, while there are additional fluctuations in the $x_{1}$ directions due to external fields or an irradiating heat bath leading to a higher effective temperature $T_{1}[26]$, see [47] for an experimental realization or [48] for an equivalent electric circuit system.

By the fluctuation-dissipation theorem, the coupling to the environments leads, in both directions, to fluctuating forces $\sqrt{2 k_{\mathrm{B}} T_{i} \gamma_{i}} \eta_{i}(t)$ on the one hand and frictional forces $-\gamma_{i} \dot{x}_{i}$ on the other hand, where $\gamma_{i}$ are the respective friction coefficients and $\xi_{i}(t)$ are independent Gaussian white-noise processes with $\left\langle\eta_{i}(t)\right\rangle=0$ and $\left\langle\eta_{i}(t) \eta_{j}\left(t^{\prime}\right)\right\rangle=\delta_{i j} \delta\left(t-t^{\prime}\right)$. The particle is confined by a parabolic potential $U(x)$ with stiffnesses $u_{1}$ and $u_{2}$ along its principal axes, which are tilted by an angle $\alpha$ with respect to the coordinate axes:

$$
U(\mathbf{x})=\frac{1}{2} \mathbf{x}^{T} \mathbf{R}_{\alpha}^{T} \mathbf{u} \mathbf{R}_{\alpha} \mathbf{x}, \quad \quad \mathbf{R}_{\alpha}=\left(\begin{array}{cc}
\cos \alpha & -\sin \alpha \\
\sin \alpha & \cos \alpha
\end{array}\right), \quad \mathbf{u}=\left(\begin{array}{cc}
u_{1} & 0 \\
0 & u_{2}
\end{array}\right) .
$$

Due to the asymmetry of the thermal and restoring forces (for $T_{1} \neq T_{2}, u_{1} \neq u_{2}$, and $\alpha \neq \pi n / 4, n \in \mathbb{Z}$ ), the particle reaches a non-equilibrium steady state and rotates around the origin on average [26]. It thereby exerts a torque on 
the environment and can thus work as a microscopic heat engine. To quantify the work done, we generalize the model studied in [26] by introducing an additional external force (see also [46])

$$
\boldsymbol{f}_{\text {ext }}(\mathbf{x})=-f_{\text {ext }} \boldsymbol{\epsilon}, \quad \text { where } \boldsymbol{\epsilon}=\left(\begin{array}{cc}
0 & 1 \\
-1 & 0
\end{array}\right)
$$

is the two dimensional antisymmetric tensor. In the overdamped limit the dynamics of the Brownian Gyrator is then described by the equations of motion

$$
\dot{\boldsymbol{x}}(t)=-\mathbf{A} \boldsymbol{x}(t)+\mathbf{B} \boldsymbol{\eta}(t),
$$

where

$$
\mathbf{A}=\left(\begin{array}{cc}
\frac{K_{11}}{\gamma_{1}} & \frac{K_{12}}{\gamma_{1}} \\
\frac{K_{21}}{\gamma_{2}} & \frac{K_{22}}{\gamma_{2}}
\end{array}\right), \quad \mathbf{K}=\mathbf{R}_{\alpha}^{T} \mathbf{u} \mathbf{R}_{\alpha}+f_{\text {ext }} \boldsymbol{\epsilon}, \quad \mathbf{B}=\left(\begin{array}{cc}
\sqrt{\frac{2 k_{B} T_{1}}{\gamma_{1}}} & 0 \\
0 & \sqrt{\frac{2 k_{B} T_{2}}{\gamma_{2}}}
\end{array}\right)
$$

For the range of parameter values where the matrix $\mathbf{A}$ is positive definite, the system reaches a steady state with probability distribution [47]

$$
p_{\text {st }}(\boldsymbol{x})=\frac{1}{2 \pi \sqrt{\operatorname{det} \boldsymbol{\Sigma}(\infty)}} \exp \left(-\frac{1}{2} \boldsymbol{x} \boldsymbol{\Sigma}^{-1}(\infty) \boldsymbol{x}\right)
$$

where $\boldsymbol{\Sigma}(\infty)$ is obtained as a solution of

$$
\mathbf{A} \boldsymbol{\Sigma}(\infty)+\boldsymbol{\Sigma}(\infty) \mathbf{A}^{T}=2 \mathbf{D}, \quad \mathbf{D}=\frac{1}{2} \mathbf{B B}^{T} .
$$

The work done by the external load force $\boldsymbol{f}_{\text {ext }}$ as well as the heat taken from the hot reservoir over a process of time duration $\tau$ can be obtained using the standard definitions of stochastic thermodynamics as

$$
W[\boldsymbol{x}(\cdot)]=\sum_{i, j} \int_{0}^{\tau} Y_{i j}^{W} x_{j} d x_{i}, \quad Q_{1}[\boldsymbol{x}(\cdot)]=\sum_{i, j} \int_{0}^{\tau} Y_{i j}^{Q_{1}} x_{j} d x_{i}
$$

with

$$
\mathbf{Y}^{W}=-\mathrm{f}^{\mathrm{ext}} \boldsymbol{\epsilon}, \quad \mathbf{Y}^{Q_{1}}=\left(\begin{array}{cc}
K_{11} & K_{12} \\
0 & 0
\end{array}\right)
$$

In Fig. 6, we display the resulting average input and output powers $\left\langle q_{1}\right\rangle,\left\langle q_{2}\right\rangle$, and $\langle w\rangle$ as a function of the load amplitude $f_{\text {ext }}$ for a certain choice of parameters. It illustrates that the system indeed works as a heat engine for moderate loads with $f_{\text {ext }}=-1 \ldots 0$.

Now using the path integral formalism, the moment generating function (MGF) of $Q_{1}$ and $W$ at arbitrary times can be obtained as

$$
\Psi_{\tau}\left(\lambda_{Q}, \lambda_{W}\right)=\left\langle e^{-\lambda_{Q} Q_{1}-\lambda_{W} W}\right\rangle_{\tau}=\int d \boldsymbol{x}_{0} p_{\mathrm{st}}\left(\boldsymbol{x}_{0}\right) \int d \boldsymbol{x}_{\tau} \int_{\boldsymbol{x}_{0}}^{\boldsymbol{x}_{\tau}} D \boldsymbol{x}[(\cdot)] P[\boldsymbol{x}(\cdot)] e^{-\lambda_{Q} Q_{1}[\boldsymbol{x}(\cdot)]-\lambda_{W} W[\boldsymbol{x}(\cdot)]}
$$

where

$$
P[\boldsymbol{x}(\cdot)] \propto \exp \left(-\int_{0}^{\tau} d t(\dot{\boldsymbol{x}}(t)+\mathbf{A} \boldsymbol{x}(t))^{T} \frac{1}{2 \mathbf{D}}(\dot{\boldsymbol{x}}(t)+\mathbf{A} \boldsymbol{x}(t))\right)
$$

denotes the Onsager-Machlup path weight [51-53]. Since all the terms in the exponent of the RHS of Eq. (29) are quadratic in $x_{1}, x_{2}$ and their derivatives, we can write this as [49]

$$
\begin{aligned}
\left\langle e^{-\lambda_{Q} Q_{1}[\boldsymbol{x}(\cdot)]-\lambda_{W} W[\boldsymbol{x}(\cdot)]}\right\rangle & =\int d \boldsymbol{x}_{0} \int d \boldsymbol{x}_{\tau} \int_{\boldsymbol{x}_{0}}^{\boldsymbol{x}_{\tau}} D \boldsymbol{x}[(\cdot)] \exp \left(\int_{0}^{\tau} \boldsymbol{x}(t) \hat{\mathbf{O}}_{\lambda_{Q}, \lambda_{W}} \boldsymbol{x}(t)+\text { Boundary terms }\right) \\
& =\sqrt{\frac{\operatorname{det} \hat{\mathbf{O}}_{0,0}}{\operatorname{det} \hat{\mathbf{O}}_{\lambda_{Q}, \lambda_{W}}}}
\end{aligned}
$$




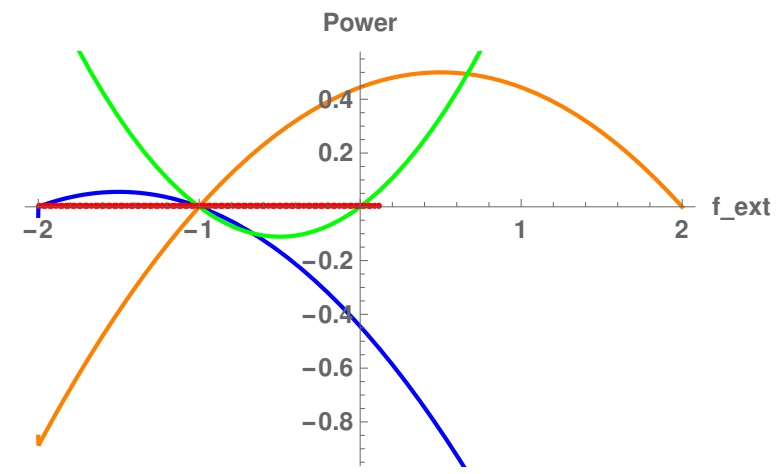

Figure 6. Average power supplied from the two reservoirs $\left(\left\langle q_{1}\right\rangle\right.$ orange, $\left\langle q_{2}\right\rangle$ blue) and by the external load force $(\langle w\rangle$ green). The solid lines correspond to the analytical solution. In the units chosen, the system reaches a steady state in the parameter range $f_{\text {ext }}=-2$ to 2 . Further, it acts as a heat pump (refrigerator, $\left\langle q_{2}\right\rangle>0,\left\langle q_{1}\right\rangle<0$ and $\langle w\rangle>0$ ) between $f_{\text {ext }}=-2$ to -1 and as a heat engine $\left(\left\langle q_{2}\right\rangle<0,\left\langle q_{1}\right\rangle>0\right.$ and $\left.\langle w\rangle<0\right)$ between $f_{\text {ext }}=-1$ to 0 . Beyond this point, we observe a trivial heat transfer from the hot to the cold reservoir. For values of $f_{\text {ext }}$ outside $f_{\text {ext }}=-2$ to 2 , the external work trivially heats both the hot and cold reservoirs, operating as a dud engine. The range of $f_{\text {ext }}=-2$ to 0.1 that is marked red corresponds to the case when the efficiency rate function $J(\eta)$ has an infinite plateau as in the top panel of Fig. 4 from the main text. In the range of $f_{\text {ext }}=0.1$ to $1, J(\eta)$ has a finite plateau as in the lower panels of Fig. 4 from the main text. Parameter values: $k_{B} T_{1}=1$, $k_{B} T_{2}=1 / 3, u_{1}=5, u_{2}=1, \alpha=\pi / 4, \gamma_{1}=\gamma_{2}=1$.

Here the operator $\hat{\mathbf{O}}$ is a matrix with differential operators as its entries [54], and the determinants that appear in Eq. (32) are functional determinants. For our problem, it can be shown that the matrix $\mathbf{O}$ has the form

$$
\hat{\mathbf{O}}=\left[\begin{array}{cc}
-a \frac{d^{2}}{d t^{2}}+b, & c \frac{d}{d t}+d \\
-c \frac{d}{d t}+d & -e \frac{d^{2}}{d t^{2}}+f
\end{array}\right]
$$

where

$$
\begin{aligned}
a & =\frac{1}{4 D_{11}} \\
b & =\frac{1}{2}\left(\frac{A_{11}^{2}}{2 D_{11}}+\frac{A_{21}^{2}}{2 D_{22}}\right) \\
c & =\frac{1}{2}\left(-\frac{A_{12}}{2 D_{11}}+\frac{A_{21}}{2 D_{22}}\right)-\lambda_{Q} \frac{A_{12}}{2}+\lambda_{W} f_{\mathrm{ext}}, \\
d & =\frac{1}{2} \frac{A_{11} A_{12}}{2 D_{11}}+\frac{1}{2} \frac{A_{21} A_{22}}{2 D_{22}} \\
e & =\frac{1}{4 D_{22}} \\
f & =\frac{1}{2}\left(\frac{A_{12}^{2}}{2 D_{11}}+\frac{A_{22}^{2}}{2 D_{22}}\right) .
\end{aligned}
$$

Then the determinant ratio that appears in Eq. (32) can be computed using a technique described in [54] and recently used in [49], which is based on the spectral- $\zeta$ functions of Sturm-Liouville type operators. Applying this method to the model at hand, it can be shown that this ratio can be obtained in terms of a characteristic polynomial function $F$ as

$$
\left\langle e^{-\lambda_{Q} Q_{1}[\boldsymbol{x}(\cdot)]-\lambda_{W} W[\boldsymbol{x}(\cdot)]}\right\rangle_{\tau}=\sqrt{\frac{F(0,0)}{F\left(\lambda_{Q}, \lambda_{W}\right)}}, \quad F\left(\lambda_{Q}, \lambda_{W}\right) \equiv \operatorname{Det}[M+N H(\tau)] .
$$

Here $H$ is the matrix of independent and suitably normalized fundamental solutions $\boldsymbol{x}^{1}(t), \ldots, \boldsymbol{x}^{4}(t)$ of the homogeneous equation $\hat{\mathbf{O}} \boldsymbol{x}=0$,

$$
H(t)=\left[\begin{array}{cccc}
x_{1}^{1}(t) & x_{1}^{2}(t) & x_{1}^{3}(t) & x_{1}^{4}(t) \\
x_{2}^{1}(t) & x_{2}^{2}(t) & x_{2}^{3}(t) & x_{2}^{4}(t) \\
\dot{x}_{1}^{1}(t) & \dot{x}_{1}^{2}(t) & \dot{x}_{1}^{3}(t) & \dot{x}_{1}^{4}(t) \\
\dot{x}_{2}^{1}(t) & \dot{x}_{2}^{2}(t) & \dot{x}_{2}^{3}(t) & \dot{x}_{2}^{4}(t)
\end{array}\right], \quad H(0)=\mathbf{I}_{4},
$$


and $M$ and $N$ contain information about the boundary conditions from Eq. (31), for which we require

$$
M\left[\begin{array}{c}
\boldsymbol{x}(0) \\
\dot{\boldsymbol{x}}(0)
\end{array}\right]=0, \quad \quad N\left[\begin{array}{c}
\boldsymbol{x}(\tau) \\
\dot{\boldsymbol{x}}(\tau)
\end{array}\right]=0 .
$$

A derivation of Eq. (35), applicable to a class of driven Langevin systems with quadratic actions, is given in [49]. We stress that the expression given in Eq. (35) is valid within the domain $C_{\lambda_{Q}}, \lambda_{W}$ for which the operator $\hat{\mathbf{O}}$ doesn't have negative eigenvalues. The MGF is not convergent outside this domain.

For the Brownian gyrator problem, we obtain the four independent solutions as

$$
\begin{aligned}
& x_{1}^{i}(t)=\exp \left( \pm t \frac{\sqrt{ \pm \frac{\sqrt{a^{2} f^{2}-2 a b e f-2 a c^{2} f+4 a d^{2} e+b^{2} e^{2}-2 b c^{2} e+c^{4}}}{a e}+\frac{b}{a}-\frac{c^{2}}{a e}+\frac{f}{e}}}{\sqrt{2}}\right), \\
& x_{2}^{i}(t)=\frac{x_{1}^{i}(t)\left(-\left(c^{2} d-a d f\right)\right)+c\left(a f-c^{2}\right) x_{1}^{i \prime}(t)-a c e x_{1}^{i \prime \prime \prime}(t)-a d e x_{1}^{i \prime \prime}(t)(t)}{b c^{2}-a d^{2}} .
\end{aligned}
$$

The matrices $M$ and $N$ are given by

$$
\begin{aligned}
& M=\left(\begin{array}{cccc}
-\frac{2 D_{11} \lambda_{Q} A_{11}+A_{11}-2 D_{11} \Sigma_{11}}{\left.4 D_{11}-\mathrm{f}_{\mathrm{ext}} \lambda_{W}\right)} & -\frac{2 D_{11} \lambda_{Q} A_{12}+A_{12}-2 D_{11} \mathrm{f}_{\mathrm{ext}} \lambda_{W}-2 D_{11} \Sigma_{12}}{4 D_{11}} & -\frac{1}{4 D_{11}} & 0 \\
-\frac{A_{21}-2 D_{22}\left(\Sigma_{22}\right.}{4 D_{22}} & -\frac{A_{22}-2 D_{22} \Sigma_{22}}{4 D_{22}} & 0 & -\frac{1}{4 D_{22}} \\
0 & 0 & 0 & 0 \\
0 & 0 & 0 & 0
\end{array}\right), \\
& N=\left(\begin{array}{cccc}
0 & 0 & 0 & 0 \\
0 & 0 & 0 & 0 \\
\frac{2 D_{11} \lambda_{Q} A_{11}+A_{11}}{4 D_{11} \mathrm{f}_{11}} & \frac{2 D_{11} \lambda_{Q} A_{12}+A_{12}-2 D_{11} \mathrm{f}_{\mathrm{ext}} \lambda_{W}}{4 D_{11}} & \frac{1}{4 D_{11}} & 0 \\
\frac{A_{21}+2 D_{22} \mathrm{exx}_{W}}{4 D_{22}} & \frac{A_{22}}{4 D_{22}} & 0 & \frac{1}{4 D_{22}}
\end{array}\right) .
\end{aligned}
$$

Using these, the MGF can be computed exactly using Eq. (35). Notice that the solution is valid for arbitrary time $\tau$. Various interesting finite time aspects of this solution will be discussed in a future publication. Here we focus on the large time limit, where the leading order form of the MGF is given by

$$
\Psi_{\tau}\left(\lambda_{Q}, \lambda_{W}\right) \sim g\left(\lambda_{Q}, \lambda_{W}\right) e^{\tau \phi\left(\lambda_{Q}, \lambda_{W}\right)} .
$$

Using the exact solution obtained from Eq. (35), the large time functional form given above can be obtained by performing an asymptotic expansion using Mathematica. We provide here the exact functional forms for the completion of the discussion:

$$
\phi\left(\lambda_{Q}, \lambda_{W}\right)=\frac{1}{8} \sqrt{\frac{D_{22}^{2}\left(16 D_{11}^{2}\left(2 a e \sqrt{\frac{b f-d^{2}}{a e}}+a f+b e\right)-A_{12}^{2}\right)+2 A_{12} A_{21} D_{11} D_{22}-A_{21}^{2} D_{11}^{2}}{a D_{11}^{2} D_{22}^{2} e}}-\sqrt{-\frac{\left(-\frac{A_{12}}{D_{11}}-2 A_{12} \lambda_{Q}+\frac{A_{21}}{D_{22}}+4 \mathrm{f}_{\mathrm{ext}} \lambda_{W}\right)^{2}}{64 a e}+\frac{1}{4} \sqrt{\frac{4 b f}{a e}-\frac{4 d^{2}}{a e}}+\frac{b}{4 a}+\frac{f}{4 e}} .
$$

In terms of the function $\Gamma\left(\lambda_{Q}, \lambda_{W}\right)$ defined as

$$
\begin{aligned}
& \Gamma\left(\lambda_{Q}, \lambda_{W}\right)= \\
& \frac{-a e}{32 a e^{2}\left(a d^{2}-b c^{2}\right) \sqrt{\frac{b f-d^{2}}{a e}}\left(2\left(a\left(2 e \sqrt{\frac{b f-d^{2}}{a e}}+f\right)+b e-c^{2}\right)\right)}\left[\sqrt{-\frac{-2 a e \sqrt{\frac{b f-d^{2}}{a e}}-a f-b e+c^{2}}{a e}}\left(4 e\left(a\left(c m s \sqrt{\frac{b f-d^{2}}{a e}}+d\left(o s \sqrt{\frac{b f-d^{2}}{a e}}+l m-n p\right)\right)+c o(b l-d p)\right)\right)+\right. \\
& \left.4\left(b\left(c\left(e\left(o s \sqrt{\frac{b f-d^{2}}{a e}}+l m-n p\right)+f o s\right)+c^{2} n s+d e l o\right)+c^{2}(-e) o p \sqrt{\frac{b f-d^{2}}{a e}}+a\left(c e(l m-n p) \sqrt{\frac{b f-d^{2}}{a e}}+d\left(e(l o+m s) \sqrt{\frac{b f-d^{2}}{a e}}-d n s+f m s\right)\right)-c^{2} d m s-c d^{2} o s-d^{2} e o p\right)\right] \\
& {\left[\sqrt{\frac{a\left(2 e \sqrt{\frac{b f-d^{2}}{a e}}+f\right)+b e-c^{2}}{a e}}\left(-4 e\left(a\left(c i y \sqrt{\frac{b f-d^{2}}{a e}}+d\left(v y \sqrt{\frac{b f-d^{2}}{a e}}+i x-u w\right)\right)+c v(b x-d w)\right)\right)+\right.} \\
& \left.4\left(b\left(c\left(e\left(v y \sqrt{\frac{b f-d^{2}}{a e}}+i x-u w\right)+f v y\right)+c^{2} u y+d e v x\right)+c^{2}(-e) v w \sqrt{\frac{b f-d^{2}}{a e}}+a\left(c e(i x-u w) \sqrt{\frac{b f-d^{2}}{a e}}+d\left(e(i y+v x) \sqrt{\frac{b f-d^{2}}{a e}}-d u y+f i y\right)\right)-c^{2} d i y-c d^{2} v y-d^{2} e v w\right)\right],
\end{aligned}
$$$$
\text { where }\left(\begin{array}{ccc}
m & n & o \\
p & l & s
\end{array}\right)=\left(\begin{array}{lll}
N_{31} & N_{32} & N_{33} \\
N_{41} & N_{42} & N_{44}
\end{array}\right), \quad\left(\begin{array}{ccc}
i & u & v \\
w & x & y
\end{array}\right)=\left(\begin{array}{lll}
M_{11} & M_{12} & M_{13} \\
M_{21} & M_{22} & M_{24}
\end{array}\right)
$$

we obtain

$$
g\left(\lambda_{Q}, \lambda_{W}\right)=\sqrt{\frac{\Gamma(0,0)}{\Gamma\left(\lambda_{Q}, \lambda_{W}\right)}} .
$$

